\title{
Analysis of Void Volume in Composite Electrode of All-solid-state Lithium-ion Battery Employing FIB-SEM and Union Operation Image Processing
}

\author{
Yuta Yamamoto $^{1}$, Yasutoshi Iriyama ${ }^{2}$, and Shunsuke Muto ${ }^{1,4}$ \\ ${ }^{1 .}$ High Voltage Electron Microscope Laboratory, Institute of Materials and Systems for Sustainability, \\ Nagoya University, Furo-cho, Nagoya, Japan \\ 2. Department of Materials, Physics, and Energy Engineering, Nagoya University, Furo-cho, Nagoya, \\ Japan \\ 3. JST-ALCA, Chiyoda-ku, Tokyo, Japan \\ 4. Advanced Measurement Technology Center, Institute of Materials and Systems for Sustainability, \\ Nagoya University, Nagoya, Japan
}

We had proposed an image-processing scheme using union operation suitable for extracting target features with hierarchical dimensions from the original data, and applied it to void analysis in a composite electrode of an all-solid-state lithium ion battery (LIB) [1]. Void analysis is very important in developing better composite electrodes for all-solid-state LIBs because internal voids should increase the interfacial resistance.

Film formation of electrode-solid electrolyte composites by the aerosol deposition (AD) method [2] has attracted attention as an effective way to improve the density of the composite electrodes [3, 4]. In the present study, three-dimensional (3D) structure of the composite electrode, which was modified by the $\mathrm{AD}$ process to reduce the internal void volume, was investigated using FIB-SEM system. The void volume in the composite electrode was then analyzed by an image-processing method based on the union operation [1].

A conventional composite electrode was composed of $\mathrm{NMC}\left(\mathrm{LiNi}_{1 / 3} \mathrm{Co}_{1 / 3} \mathrm{Mn}_{1 / 3} \mathrm{O}_{2}\right)$ electrode and oxidebased lithium-conductive solid electrolytes, Li-Al-Ti-P-O glass ceramics (LATP) manufactured by Ohara Inc., which exhibits the highest lithium conductivity $\left(10^{-3} \mathrm{~S} \mathrm{~cm}^{-1}\right.$ at $\left.298 \mathrm{~K}\right)$ [5]. This composite was prepared on a silicon substrate by AD. Another modified composite electrode was composed of LNM ( $\left.\mathrm{LiNi}_{0.5} \mathrm{Mn}_{1.5} \mathrm{O}_{4}\right)$ electrode and LATP solid electrolyte. This composite was prepared on a platinum substrate by $\mathrm{AD}$, then heat-treated at $500{ }^{\circ} \mathrm{C}$

A FIB-SEM system (MI4000L, HITACHI) having an orthogonal geometry was used for 3D structural investigation by automatic alternating operations of SEM observation and FIB cross-sectioning. The SEM system was operated at an accelerating voltage of $15 \mathrm{kV}$ for secondary electron (SE) observation using the annular in-lens SE detector of 'Gemini column" TM'. All the SEM images were taken at 1000 pixel $^{2}$ at a resolution of $20 \mathrm{~nm}^{2}$ pixel $^{-2}$. FIB cross-sectioning were done in 1,000 steps at a width of 20 nm.

The software, Avizo fire (FEI ${ }^{\mathrm{TM}}$ Company) was used for 3D image reconstruction, image processing and volume rendering. All the SEM images subsequently obtained was aligned in position with the substrates and vacuum spaces trimmed away, and then three different objective filters was separately applied: a median blur filter to filter out relatively larger voids, a morphological opening filter for small dot-shaped voids and a morphological closing filter for small voids with concave contrasts. The processed three filtered image data were individually subtracted from the original volume data with 
additional structures other than voids carefully ruled out by setting appropriate intensity thresholds. The resultant three volume data were finally unified by the union operation.

Figure 1(a) shows a bird's eye view of the composite electrode fabricated by the conventional method. The electrode and the solid electrolyte correspond to the brighter and darker contrasts respectively, exhibiting the layer structure. Figure 1(b) shows the 3D configuration of voids in (a). Voids were distributed over the entire composite. On the other hand, Figures 1(c) and (d) are respectively the counterparts of (a) and (b) for the modified composite electrode. The total volume fraction of the modified composite (Figure 1(d)) was estimated to be 0.036 vol.\%, whereas that of the conventional composite (Figure 1(b)) was $0.15 \mathrm{vol} \%$. It is clearly shown that by modifying the fabrication process the density of the composite electrode was significantly improved.

\section{References:}

[1] Yuta Yamamoto, Yasutoshi Iriyama and Shunsuke Muto, Microscopy 65 (2016), p. 191.

[2] Jun Akedo J. Am. Ceram. Soc. 89 (2006), p. 1834.

[3] Shinya Iwasaki et al, J. Power Sour. 272 (2014), p. 1086.

[4] Takeshi Kato et al, J. Power Sour. 303 (2016), p. 65.

[5] Jie Fu, J. Am. Ceram. Soc. 80 (1997), p. 1901.
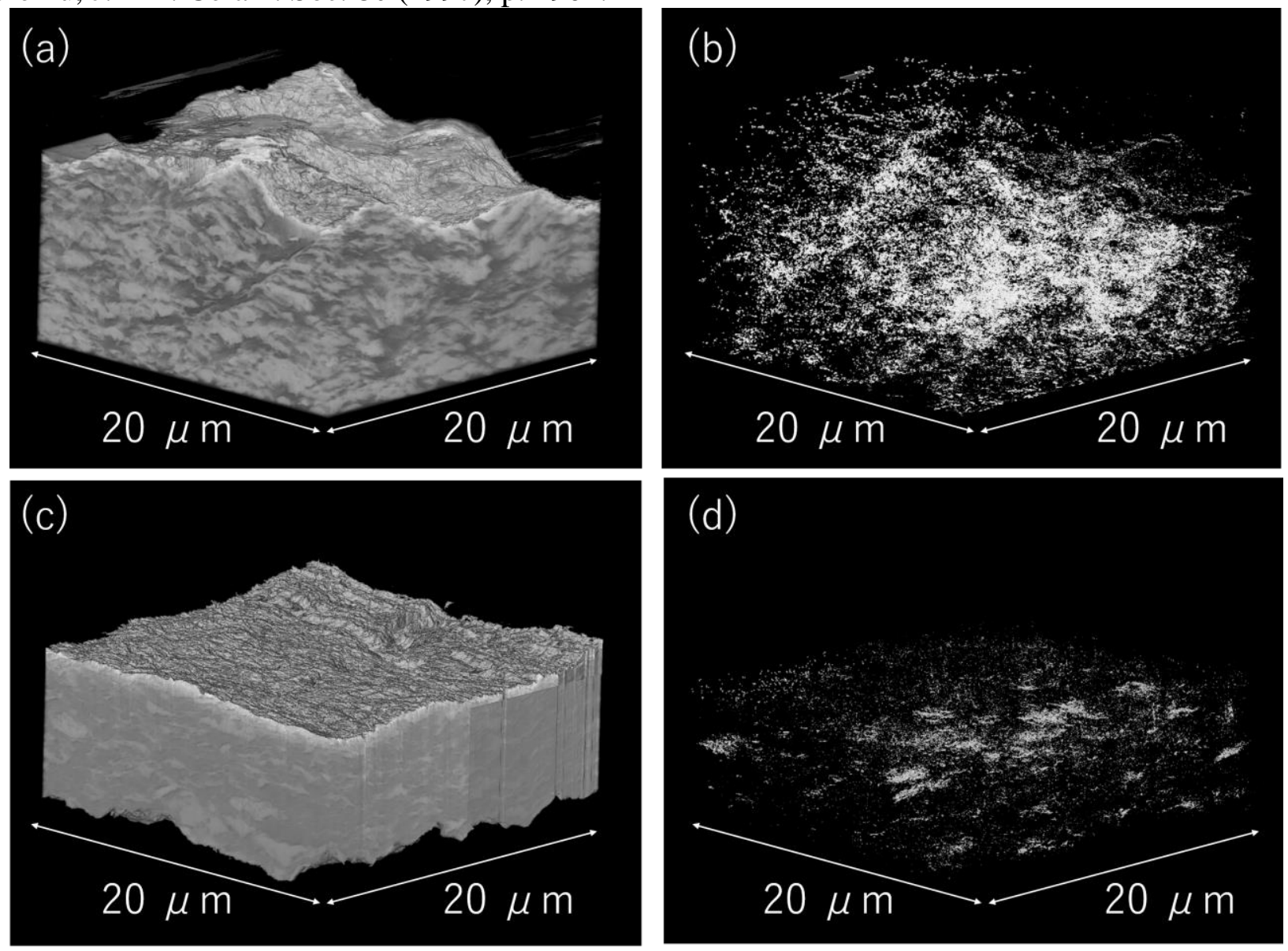

Figure 1. (a) Bird's eye view of the composite electrode of NMC - LATP by the previous method. (b) 3D configuration of the voids inside (a). (c) Bird's eye view of the composite electrode of LNM - LATP after heat treatment, by the present modified method. (d) 3D configuration of the voids inside (c). 